\title{
Error-related anterior cingulate cortex activity and the prediction of conscious error awareness
}

\author{
Catherine Orr* and Robert Hester \\ Department of Psychological Sciences, University of Melbourne, Melbourne, VIC, Australia
}

\author{
Edited by: \\ Tilmann A. Klein, Max Planck \\ Institute for Human Cognitive and \\ Brain Sciences, Germany \\ Reviewed by: \\ Michael X. Cohen, University of \\ Amsterdam, Netherlands \\ Simon van Gaal, University of \\ Amsterdam, Netherlands

\section{*Correspondence:} \\ Catherine Orr, Department of \\ Psychological Sciences, University \\ of Melbourne, Melbourne, VIC 3010, \\ Australia. \\ e-mail: corr@unimelb.edu.au
}

\begin{abstract}
Research examining the neural mechanisms associated with error awareness has consistently identified dorsal anterior cingulate cortex (ACC) activity as necessary but not predictive of conscious error detection. Two recent studies (Steinhauser and Yeung, 2010; Wessel et al., 2011) have found a contrary pattern of greater dorsal ACC (dACC) activity [in the form of the error-related negativity (ERN)] during detected errors, but suggested that the greater activity may instead reflect task influences (e.g., response conflict, error probability) and or individual variability (e.g., statistical power). We re-analyzed fMRI BOLD data from 56 healthy participants who had previously been administered the Error Awareness Task (EAT), a motor Go/No-go response inhibition task in which subjects make errors of commission of which they are aware (Aware errors), or unaware (Unaware errors). Consistent with previous data, the activity in a number of cortical regions was predictive of error awareness, including bilateral inferior parietal and insula cortices, however, in contrast to previous studies, including our own smaller sample studies using the same task, error-related dACC activity was significantly greater during aware errors when compared to unaware errors. While the significantly faster RT for aware errors (compared to unaware) was consistent with the hypothesis of higher response conflict increasing ACC activity, we could find no relationship between dACC activity and the error RT difference. The data suggests that error awareness is associated with error-related dACC activity but that the role of this activity is probably best understood in relation to the activity in other regions. Activity in the dACC may be important to conscious error detection, but it remains unclear what task and individual factors influence error awareness.
\end{abstract}

Keywords: error awareness, anterior cingulate cortex, performance monitoring

\section{INTRODUCTION}

Goal directed behavior requires the ability to recognize appropriate responses and to flexibly adjust behavior in response to an error. Even in the absence of explicit feedback, people demonstrate characteristic reactions following an error and often spontaneously correct their response (Nieuwenhuis et al., 2001; O'Connell et al., 2009). These behaviors suggest the activity of a performance monitoring system, which evaluates actions and allows adaptive adjustments in attention and cognitive control mechanisms to reduce the likelihood of repeating an error (Ullsperger and von Cramon, 2001). Cognitive neuroimaging research has consistently implicated the prefrontal cortex (PFC) and the anterior cingulate cortex (ACC) in error processing (Garavan et al., 2003; Ridderinkhof et al., 2004). Moreover, hypoactivity in this network has been associated with deficits in error-related processing and subsequent behavioral adjustments observed in populations with schizophrenia (Morris et al., 2006), Attention Deficit Hyperactivity Disorder (ADHD; BurgioMurphy et al., 2007), Alzheimer's disease (Mathalon et al., 2003), and substance use disorders (Franken et al., 2007).

It has been suggested that error processing can proceed, at least in part, outside of conscious awareness. We are not aware of every error we commit and research explicitly assessing error awareness has shown that even spontaneously corrected errors can go unreported (Endrass et al., 2005, 2007; Wessel et al., 2011). Although some post-error adaptations can occur outside of awareness, Ullsperger and colleagues (2010) argued that conscious awareness is critical to the affective significance of an error. It is likely that the affective evaluation of one's performance influences overall motivation and longer-term strategic approach to the task. Reduced awareness of errors has been associated with a lack of insight into maladaptive behaviors in drug addiction (Hester et al., 2007), ADHD (O'Connell et al., 2009), and psychopathy (Brazil et al., 2009). It is important, therefore, to understand the conditions under which errors reach consciousness and the neural correlates of error awareness.

Electrophysiological research has consistently associated a positively deflecting event-related potential (ERP) observed 100-200 ms following an error (the error positivity: Pe) with awareness of the error (Endrass et al., 2005; Overbeek et al., 2005). Typically, the Pe is reduced or absent for those errors of which the participant remains subjectively unaware. It has been suggested that the Pe may be a context specific P3, a component associated with attentional orienting to stimuli of motivational significance (Ridderinkhof et al., 2009). The P3/Pe is believed to arise from activity in the prefrontal and parietal cortices (Soltani and Knight, 
2000), and is recorded as an average over a centro-parietal electrode site. Functional neuroimaging research corroborates these suggestions in implicating a network of frontal and parietal regions in error awareness, notably the insulae (Hester et al., 2005, 2009a; Klein et al., 2007), bilateral inferior parietal (Hester et al., 2005, 2009a), and bilateral mid frontal (Hester et al., 2005, 2009a) cortices.

Performance monitoring is also associated with a negatively deflecting ERP that occurs immediately following the erroneous response, the error-related negativity (ERN) (Falkenstein et al., 1991), which is believed to reflect a stage of error processing that is functionally distinct from that reflected in the Pe (Overbeek et al., 2005). For example, although the ERN is significantly larger following an error than a correct response (Dehaene et al., 1994), ERN magnitude does not reliably discriminate aware from unaware errors (Endrass et al., 2005; O'Connell et al., 2007; but cf. Scheffers and Coles, 2000 and refer Wessel in this issue for a comprehensive review). It has been suggested that the ERN represents encoding of an element of the task environment or of task performance associated with errors; specifically, it has been proposed that the ERN reflects response conflict (Botvinick et al., 2001; Yeung et al., 2004), thwarted reward expectations (Holroyd et al., 2004), or detected changes in error likelihood (Brown and Braver, 2005).

The ERN has been localized to the ACC (Halgren et al., 2002; Debener et al., 2005; Wang et al., 2005) and fMRI research is largely consistent with the ERP findings in showing that, although BOLD activity in dorsal ACC (dACC) is greater for errors than correct responses, this region is not sensitive to error awareness (Hester et al., 2005, 2009a; Klein et al., 2007). The role of the ERN/dACC in error processing is unclear, but elevated levels of dACC activity have been associated with error-related autonomic arousal (Critchley et al., 2005) and with more conservative responding following an error, measured as post-error slowing (PES) of reaction time (RT) (Garavan et al., 2002; Kerns et al., 2004). Autonomic arousal and PES have been shown to be reduced or absent following unaware errors (Nieuwenhuis et al., 2001; Endrass et al., 2007; O'Connell et al., 2009). It is “surprising" (Klein et al., 2007, p. 1779), therefore, that those studies that have explicitly examined the role of dACC in error awareness have consistently reported equivalent levels of dACC activity for aware and unaware errors in healthy controls.

In contrast to the predominance of past research, two recent studies (Steinhauser and Yeung, 2010; Wessel et al., 2011) have reported sensitivity in dACC to error awareness in the form of greater ERN magnitude for aware than unaware errors. While it appears that these recent findings contradict the extensive literature on the role of the ERN/dACC, in this issue Wessel reviews past research and argues that the ERN/dACC is critically involved in error awareness. Wessel discusses findings of error sensitivity in the ERN (Maier et al., 2008) and the relationship between the ACC and awareness in other paradigms (Dehaene et al., 2003; Mayr, 2004) and suggests that methodological considerations may have obscured the role of ERN in error awareness in past research. Wessel argues that those studies that limit the time participants have to acknowledge an error, or in which signaling an error requires an additional "error awareness" response may induce a conservative response bias in which some liminal errors go unreported (cf. Nieuwenhuis et al., 2001; Woodman, 2010; Wessel et al., 2011). Alternatively, it was suggested that the inconsistent ERN/error awareness relationship might be the result of low statistical power in the reported studies. After reviewing past research, Wessel and colleagues (2011) concluded that it is common for studies to demonstrate numerically larger ERN for aware than for unaware errors (e.g., Endrass et al., 2007; Hughes and Yeung, 2011), but that this effect falls short of statistical significance. The authors proposed that small sample sizes and low numbers of unreported errors in past research mean that conclusions that the ERN is not sensitive to error awareness may have been drawn from research with insufficient statistical power.

Review of the fMRI research suggests that these studies are also vulnerable to the criticism that they may have lacked sufficient statistical power to determine the presence or absence of a relationship between the dACC and error awareness. Due to the practicalities of collecting fMRI data, sample sizes are comparatively small and experimental sessions are typically of a length that limits the number of unreported errors. As with the electrophysiological findings relating to the ERN, fMRI studies have consistently reported numerically larger BOLD signal change in the dACC for aware than unaware errors (Hester et al., 2005, 2009a; Klein et al., 2007). The fact that this difference has failed to reach statistical significance may be due, as was argued to be the case for ERP research (Wessel et al., 2011), to a lack of statistical power. Alternatively, a recent investigation using multimodal neuroimaging techniques (Agam et al., 2011) suggested that the ERN may originate in the posterior cingulate cortex (PCC), raising the possibility of a functional dissociation between the ERN and activity in the dACC. In light of these suggestions, the relationship between the dACC, erroneous responses, and error awareness warrants reinvestigation.

The aim of the present study was to determine whether more powerful statistical analyses would demonstrate a relationship between the dACC and error awareness. The present study combined the samples of three previous studies (Hester et al., 2005, 2009a, 2012) to form a group of 56 healthy controls. The studies employed the Error Awareness Task (EAT: Hester et al., 2005), a motor $\mathrm{Go} / \mathrm{No}$-go task modified to provide a measure of error awareness and to optimize unaware errors. The behavioral performance and cortical activation levels of the composite sample were analyzed to reassess the neural mechanisms associated with error awareness and error-related behavior.

\section{MATERIALS AND METHODS SUBJECTS AND TASK DESIGN}

Fifty-six right-handed participants (seven female, mean age 27, range: 20-41), reporting no history of neurological or psychological impairment, completed a version of the EAT (Hester et al., 2005). The experimental procedure was approved by the ethics committees of the relevant institutions (University of Melbourne, Trinity College Dublin, and University of Queensland), and participants provided written, informed consent at the beginning of the experimental session. The EAT is a motor Go/No-go response inhibition task, modified to allow participants to acknowledge errors of commission of which they are aware (refer Figure 1). 


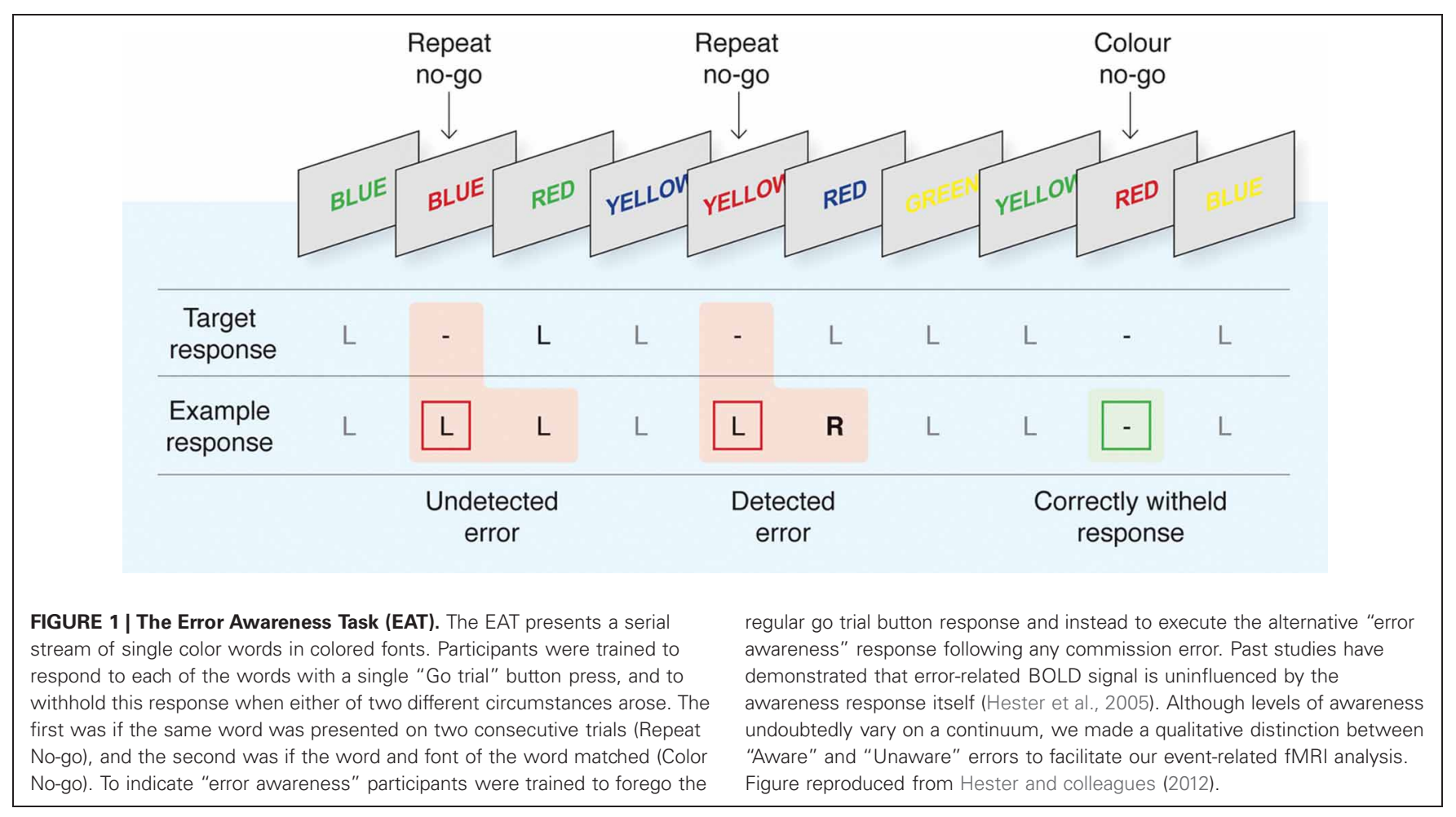

Color names, printed in colored font, were presented in a serial stream. The three samples completed versions of the task that differed slightly in the stimulus presentation and inter-stimulus interval duration, 900/600 ms (Hester et al., 2005, 2009a) or $800 / 700 \mathrm{~ms}$ (Hester et al., 2012). Participants were instructed to respond to the presentation of each stimulus with a button press (a "Go" response) unless the stimulus was a "lure," in which case they were to withhold their response ("No-go" response). Lures could take two forms: a "Repeat lure" in which the same word was presented on two consecutive trials; and a "Color lure" defined by the congruence of the color name and the font color. Color lures were defined by incongruence between the color word and the font in the two earlier studies (Hester et al., 2005, 2009a); and by congruence in the third study (Hester et al., 2012). Adopting competing inhibition rules exploits the different strengths of the stimulus-response relationships such that the overlearned behavior of reading a word would make the Repeat rule more salient than the Color rule. Previous research has suggested that this may cause the Color rule to be suppressed, producing more Color errors than Repeat errors and potentially affecting participants' awareness of the errors (Hester et al., 2005, 2009a). Participants were instructed to execute an alternative "error awareness" response on the "Go" trial following an error.

The 2005 and 2009 studies presented five blocks of 225 trials during fMRI data acquisition with lure trials pseudo randomly distributed across the 1125 experimental trials. The 2012 study presented six blocks of 225 trials. There were minor differences to the ratio of No-go: Go trials across the three experiments, with 128 lures presented in the earlier study (Hester et al., 2005) and 125 and 150, respectively, in the two later studies (Hester et al., 2009a, 2012). On average, a lure was presented every 8.95 trials, corresponding to an average inter-lure interval of $13.42 \mathrm{~s}$. While some other variations in the design of these tasks existed, only the commission errors made during the aforementioned design were considered, with the assumption made that the event-related analysis would minimize the influence of unrelated task variance.

\section{SCANNING PARAMETERS AND DATA ANALYSES}

Scanning for the original study (Hester et al., 2005) was conducted using contiguous $5 \mathrm{~mm}$ sagittal slices covering the entire brain from a $1.5 \mathrm{~T}$ Siemens Vision scanner using a single shot, $2^{*}$-weighted echo-planar pulse sequence $(\mathrm{TE}=50 \mathrm{~ms}$; $\mathrm{TR}=2000 \mathrm{~ms} ; \mathrm{FOV}=256 \mathrm{~mm} ; 64 \times 64$ matrix $).$ High resolution T1-weighted structural MPRAGE images $(F O V=256 \mathrm{~mm}$, thickness $=1.0 \mathrm{~mm}$ isotropic with no gap) were acquired prior to functional imaging to allow subsequent activation localization and for spatial normalization. Stimuli were delivered using an IFIS-SA stimulus-delivery system (MRI Devices Corp., Waukesha, Wisconsin), which was equipped with a head-coil-mounted $640 \times 480$ LCD panel. This shielded LCD screen is mounted on the head-coil, directly in the subjects' line of vision. Foam padding was used to limit head movements within the coil.

Scanning for the second study (Hester et al., 2009a) was conducted using a 3T Philips Achieva scanner in which foam padding was used to restrict head movements. Contiguous $3.5 \mathrm{~mm}$ sagittal slices covering the entire brain were collected using a gradient-echo echo-planar imaging $(\mathrm{EPI})$ sequence $(\mathrm{TE}=35 \mathrm{~ms}$; $\mathrm{TR}=2000 \mathrm{~ms} ; \mathrm{FOV}=224 \mathrm{~mm}$ ). High resolution T1-weighted structural MPRAGE images (FOV $=256 \mathrm{~mm}$, isotropic $0.9 \mathrm{~mm}$ voxels) were acquired following functional imaging to allow subsequent activation localization and spatial normalization. Stimuli 
were back-projected onto a screen at the subject's feet and were viewed with the aid of prism glasses attached to the inside of the radio-frequency head-coil.

Scanning for the third study (Hester et al., 2012) using a whole-body 1.5 Tesla Siemens scanner with a gradient-echo EPI sequence. EPI images were acquired using a gradient-echo pulse sequence and sequential slice acquisition $\left(T_{R}=2000 \mathrm{~ms}\right.$, $T_{E}=30 \mathrm{~ms}$, flip angle $=90^{\circ}, 29$ contiguous slices of $3 \mathrm{~mm}$ thickness, $10 \%$ gap, in-plane resolution of $3.6 \times 3.6$ pixels in a FOV of $384 \mathrm{~mm}$ ). Activation data were registered to high resolution T1weighted isotropic $\left(1 \mathrm{~mm}^{3}\right)$ structural MPRAGE images. Only the data from the placebo condition of this study was used for this reanalysis.

Behavioral data from each participant were used to categorize the trial events into successful responses (stops), aware errors, and unaware errors. All analyses were conducted using AFNI software (http://afni.nimh.nih.gov/afni/) (Cox, 1996). Following image reconstruction, the time-series data were motion-corrected using 3D volume registration (least-squares alignment of three translational and three rotational parameters). Activation outside the brain was removed using edge detection techniques.

Separate haemodynamic impulse response functions (IRFs) at $2 \mathrm{~s}$ temporal resolution were calculated using deconvolution techniques for aware errors, unaware errors, and stop events. Response functions for all regressor events were initiated at image acquisition onsets because the presentation of all epochsof-interest was timed to coincide with the beginning of the $2 \mathrm{~s}$ TR-cycle. A non-linear regression program determined the best-fitting gamma-variate function for these IRFs as previously described (Murphy and Garavan, 2005). The area under the curve of the gamma-variate function was expressed as a percentage of the area under the baseline. The baseline in this design is indicative of task-related go trial processing that remains after the variance related to the other types of events have been removed.

The percentage area (event-related activation) map voxels were re-sampled at $1 \mathrm{~mm}^{3}$ resolution, then spatially normalized to standard MNI space (MNI 152 template), and spatially blurred with a $3 \mathrm{~mm}$ isotropic root mean squared Gaussian kernel. Group activation maps for errors were determined with one-sample $t$-tests against the null hypothesis of zero event-related activation changes (i.e., no change relative to baseline). Significant voxels within group maps passed a voxelwise statistical threshold $(t=5.23, p \leq 0.00001)$ and were required to be part of a larger $84 \mu \mathrm{l}$ cluster of contiguous significant voxels. By using a combination of probability thresholding and cluster thresholding, the aim is to maximize the power of the statistical test while holding the likelihood of false-positives to a minimum. To determine the cluster threshold we use a program called $3 \mathrm{dClustSim}$. The program is provided with the number of voxels in the group map, the spatial correlation of voxels (must be contiguous on three sides), and the voxelwise threshold. The program then runs a series of Monte Carlo simulations (10000 iterations for our study) to determine the frequency of clusters of varying sizes produced by chance. From this frequency distribution, we then select the cluster size ( $84 \mu \mathrm{l}$ given our parameters) that occurs less than $1 \%$ of the time by chance, to give a threshold of $p=0.01$ (corrected).
The activation clusters from whole-brain analyses of aware and unaware errors were used to create an OR map for the purposes of an ROI analysis. An OR map includes the voxels of activation indicated as significant from either of the constituent maps, which are presented in Appendix. The mean activation for clusters in the combined error map was then calculated for the purposes of an ROI analysis, deriving mean activation levels for aware and unaware errors, that were compared using repeated measures $t$-tests, corrected via a modified Bonferroni procedure for multiple comparisons (Keppel, 1991).

\section{RESULTS}

Performance indices for the EAT are summarized in Table $\mathbf{1 .}$ Participants correctly withheld their responses on $52.8 \%$ of the No-go trials, with significantly more successful inhibitions for Color than for Repeat lures (62.6 vs. $42.9 \%: t_{(55)}=9.76$, $p<0.001)$. Participants reported awareness of $82.5 \%$ of their errors (range 15-99\%), with a significantly greater proportion of Color errors endorsed than Repeat errors (85.5 vs. $77.2 \%$ : $\left.t_{(55)}=2.907, p<0.006\right)$. Participants' awareness of errors was not related to their inhibition accuracy $(r=-0.0115$, ns). The speed of commission error responses was significantly related to awareness of the error $\left[F_{(2,110)}=7.222, p<0.002, \eta_{p}^{2}=0.116\right]$. Post-hoc comparisons demonstrated that this effect was due to significantly faster responses for aware errors of commission than for either unaware errors or for correct "Go" responses $\left[t_{(55)}=3.403, p<0.002\right.$ and $t_{(55)}=2.680, p<0.01$, respectively].

Behavioral adjustments following No-go trials were assessed by calculating the difference between RT for the trials that immediately preceded and succeeded the lure. Participants demonstrated significant speeding of responses following aware errors $\left[t_{(55)}=10.519, p<0.001\right]$. This finding is inconsistent with other reports, in which participants slow their rate of responding following an error (Rabbitt, 1966) and this PES is exacerbated by error awareness (Nieuwenhuis et al., 2001). Reductions in RT following aware errors have consistently been reported using

Table 1 | Behavioral performance: inhibition accuracy and reaction time on the EAT.

\begin{tabular}{lc}
\hline Category & Mean (SD) \\
\hline INHIBITION ACCURACY (\%) & \\
Color lure & $42.9(23.6)$ \\
Repeat lure & $62.6(21.9)$ \\
ERROR AWARENESS (\%) & \\
Color lure & $85.5(17.7)$ \\
Repeat lure & $77.2(19.9)$ \\
REACTION TIME (ms) & \\
Go trial & $488.2(14.93)$ \\
Aware error & $479.1(16.99)$ \\
Unaware error & $535.7(22.88)$ \\
POST-LURE ADJUSTMENT (POST-LURE RT-PRE-LURE RT) \\
Correct inhibition & $-118.1(100.90)$ \\
Aware error & $-124.0(88.24)$ \\
Unaware error & $27.3(81.14)$
\end{tabular}


the EAT and it is possible that the post-error speeding observed in this paradigm is due to participants learning that lures were widely spaced. This suggestion is consistent with the significant decrease in RT for the Go trial following a correct inhibition $\left[t_{(29)}=118.124, p<0.001\right]$, but is confounded by the fact that following aware errors, participants will execute the "awareness" response without the need to process the post-lure stimulus. PES was observed following errors of which the participant remained unaware $\left[t_{(55)}=2.517, p<0.02\right]$. In order to verify that this effect was not due to strategic slowing of RT with increasing temporal distance from the last perceived lure, a surrogate data-set was generated by selecting Go trials that occurred at the same point following a consciously perceived lure as the unaware error trial. When comparing RT for those trials that immediately preceded and succeeded the surrogate trials, no significant difference was observed $\left[t_{(38)}=0.53, \mathrm{~ns}\right]$, suggesting that post-unaware error slowing was not due to changes in response strategy across trials.

The event-related functional analysis of aware and unaware errors identified eight clusters that showed significantly greater increases in activity associated with aware errors than unaware errors (Table 2). Consistent with previous reports, we observed error awareness sensitivity in clusters in the bilateral insula cortices (Figure 2C) and the IPL (Figure 2B). We also revealed activity in midline structures (dACC, pre-SMA, and SMA; Figure 2A) that discriminated aware from unaware errors ${ }^{1}$. When repeating the analysis on a subset of participants who endorsed an equivalent number of color and repeat lure errors $\left[t_{(31)}=2.01, \mathrm{~ns}\right]$, activity in all eight of the clusters identified in the full sample continued to show greater levels of BOLD activity associated with aware than with unaware errors.

Although dACC activity was sensitive to error awareness, there was no relationship between dACC activity and behavioral measures typically associated with error awareness. The speed with which errors of commission were executed was

${ }^{1}$ Note that, although the center of mass of activity in these midline structures is reported as falling in the left hemisphere, the lateral extent of these clusters was such that the bilateral dACC, pre-SMA, and SMA can be considered to discriminate between aware and unaware errors. not associated with the degree of increase in dACC activity for either aware $(r=-0.04, \mathrm{~ns})$ or unaware $(r=-0.047$, ns) errors. Nor were post-error RT adjustments correlated with the change in dACC activity for either aware $(r=-0.064$, ns) or unaware $(r=-0.007$, ns) errors. The magnitude of the discrepancy between BOLD activity in the dACC associated with aware errors and that associated with unaware errors was not related to the speed with which erroneous responses were made or the post-error adjustments in RT.

For aware errors, the relative speed of the erroneous response was related to activity in the SMA $(r=0.309, p<0.03)$, such that slower aware error RT (relative to average Go trial RT) was associated with higher levels of activity in this region. Following an aware error, the post-error adjustment in RT (post-error RTpre-lure RT) was negatively correlated with activity in the R-IPL $(r=-0.277, p<0.04)$ and with both clusters centered on the L-IPL $\left(r=-0.312, p<0.02^{2}\right.$ and $\left.r=-0.330, p<0.02^{3}\right)$, such that greater levels of activity in these regions predicted greater speeding of RT on the post-error trial. Unaware error RT and post-unaware-error RT were not correlated with BOLD activity in any of the functionally defined ROI. For none of the ROI did the magnitude of the discrepancy between BOLD activity associated with aware errors or that associated with unaware errors correlate with the speed with which erroneous responses were made or the post-error adjustment in RT.

\section{DISCUSSION}

The current study was designed to reassess the relationship between dACC activity and error awareness in light of recent reports that an electrophysiological measure of dACC activity (ERN) discriminated aware from unaware errors (Steinhauser and Yeung, 2010; Wessel et al., 2011). In addition to regions previously implicated in error awareness when assessed using the EAT, the insula and the bilateral IPL (Hester et al., 2005, 2009a), we demonstrated that aware errors were associated with significantly greater BOLD signal change in the dACC and supplementary motor cortex (SMC) than unaware errors. Although

\footnotetext{
${ }^{2}$ Center of Mass MNI co-ordinates: $x=-49, y=-34, z=50$.

${ }^{3}$ Center of Mass MNI co-ordinates: $x=-51, y=-47, z=40$.
}

Table 2 | Regions that showed significantly greater BOLD signal change for aware than unaware errors.

\begin{tabular}{|c|c|c|c|c|c|c|c|}
\hline Structure & $p$ & Vol. $\mu \mathrm{I}$ & Brodmann Area & HS & \multicolumn{3}{|c|}{ Center of mass (MNI Coordinates) } \\
\hline Inferior parietal & $<0.0007$ & 2758 & 40 & $\mathrm{R}$ & 49 & -49 & 40 \\
\hline Inferior parietal/SMG & $<0.0004$ & 1135 & 40 & L & -51 & -47 & 40 \\
\hline $\mathrm{dACC}$ & $<0.0006$ & 1510 & 32 & L & -1 & 19 & 39 \\
\hline MidCingulate/pre-SMA & $<0.01$ & 216 & 6 & L & -3 & -14 & 52 \\
\hline SMA & $<0.0004$ & 107 & 6 & L & 0 & -3 & 61 \\
\hline
\end{tabular}

Positive values for $x, y$, and $z$ coordinates denote, respectively, locations that are right, posterior, and superior relative to the anterior commissure. Significance test results indicate areas of increased activation associated with aware relative to unaware errors assessed using t-test. 


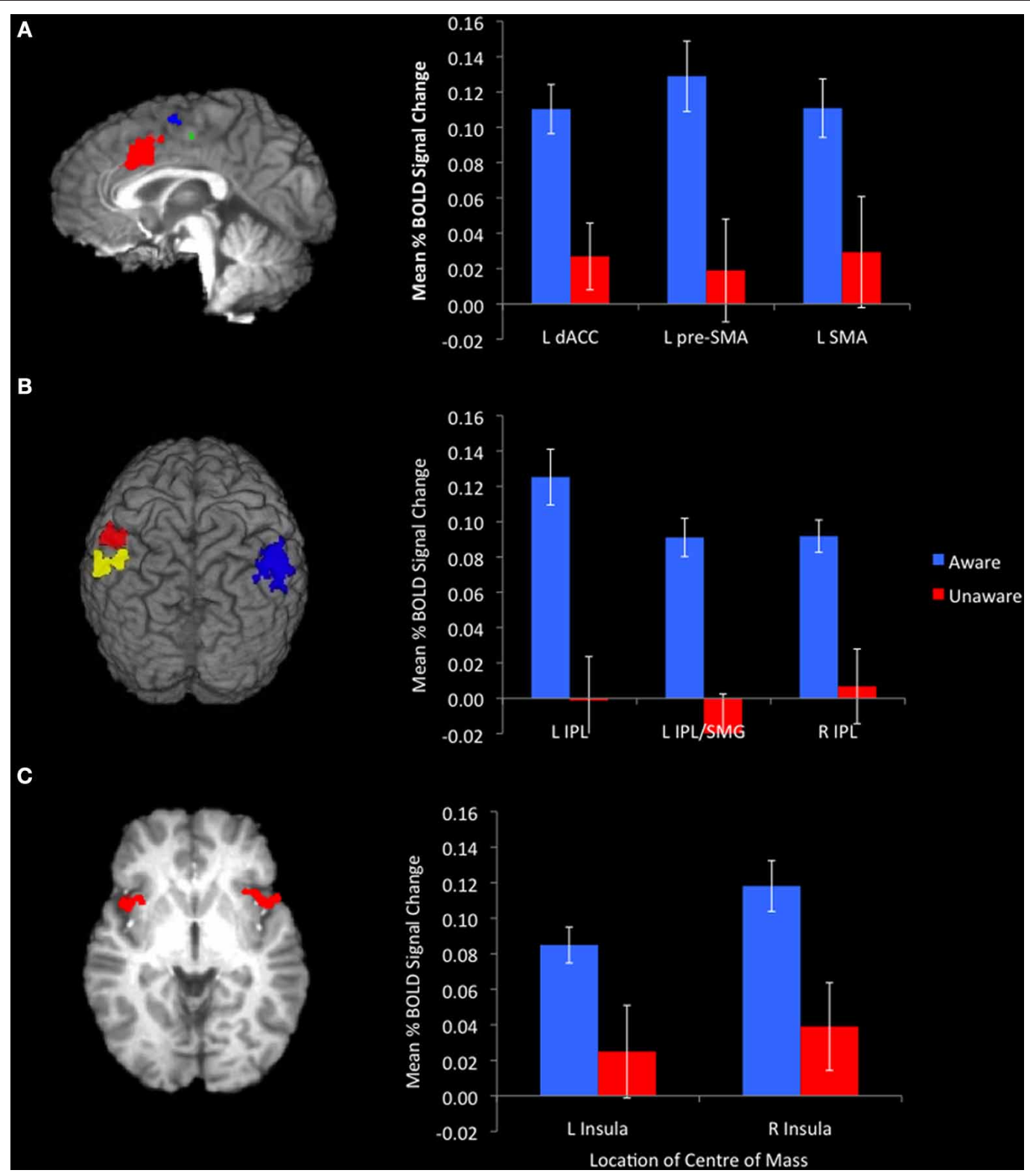

FIGURE 2 | Awareness of errors was associated with significantly greater activation in midline structures. [(A) sagittal slice at $x=-1$. Red cluster centered on the dACC, blue cluster centered on the SMA, green cluster centered on the pre-SMA)], the bilateral IPL [(B) red cluster centered on the L-IPL, yellow cluster centered on the L-IPL/SMG, blue cluster centered on the R-IPL], and insulae [(C) axial slice at $z=0$ ]. Bar graphs represent mean \%BOLD signal change relative to baseline for aware and unaware errors. the dACC showed error sensitivity, there was no direct correlation between error-related activity in this region and behaviors typically associated with error awareness (error RT and posterror RT). For this reason, and because this awareness sensitivity was only observed when reanalyzing a composite sample of 56 participants, we suggest that the relationship between dACC, error awareness, and error-related behaviors is indirect and best understood in relation to the activity in other brain regions.

An indirect relationship between dACC and error-related behaviors is consistent with the predictions of a model recently described by Holroyd and Yeung (2011). The authors suggested that the dACC is involved in the selection and evaluation of appropriate sequences of actions directed to attaining a particular goal (Botvinick et al., 2009), rather than the constituent actions themselves. For example, error-related activity in the dACC may index competition between multiple response strategies or the negative reinforcement of an unsuccessful strategy. If activity in the dACC is related to long term goal attainment, rather than the value of individual responses, it is possible that it reflects an aggregation of performance relevant information such as motivational significance, response selection parameters, and the 
efficient balance between speed and accuracy. In the context of error processing, activity in the dACC may need to surpass a certain threshold (Yeung et al., 2004) for awareness and post-error adjustments to occur. In this way, activity in the dACC would covary with error commission and contribute to the likelihood that an error will reach awareness, but may not be sufficient to produce awareness in isolation.

Increased activity in the insula cortex has consistently been associated with error awareness (Hester et al., 2005, 2009a; Klein et al., 2007), an effect that is likely due to the central role of the insula in interoceptive awareness (Critchley et al., 2004; Craig, 2009). The insula is implicated in the regulation and awareness of autonomic responses (Critchley et al., 2000; Craig, 2002) and error-related activity in the autonomic nervous system (ANS) is greater following an aware than an unaware error (O'Connell et al., 2007, 2009). There is evidence that dACC provides topdown input to systems regulating autonomic activity (Critchley et al., 2001, 2002) and that the resulting visceral sensation is perceived as a "feeling," indexed by insula activity (Critchley, 2005). Craig (2009) described this relationship as the ACC signal providing a measure of the motivational significance of an event, which is interpreted in the insula in the context of information about social and cognitive conditions to produce awareness of an event and the actor's subjective experience of it. This model is consistent with the suggestion that error-related activity in the dACC may feedforward into regions directly responsible for error awareness (Wessel et al., 2011).

The relationship between the AACC, ANS response, and the insula provides a possible means by which activity in the dACC could mediate error awareness. It is unclear, however, what is communicated by the dACC signal. One influential hypothesis argues that dACC activity indexes conflict or dissonance, in the form of a mismatch between two possible response mappings (Coles et al., 2001) or as conflict between the executed response and the correct response (Yeung et al., 2004). In any task, a proportion of errors may occur due to pre-emptive responding; it has been argued that these errors will be higher in conflict than those errors that occur due to loss of attentional set or impaired stimulus perception as the still evolving representation of the correct response will conflict with the executed response (Yeung et al., 2004). These errors will, obviously, be associated with comparatively fast RT but may also be more likely to be reported as participants become aware of both the executed response and the correct response (Scheffers and Coles, 2000; Shalgi et al., 2007; Steinhauser and Yeung, 2010). If ACC activity indexes high conflict trials, we might predict a relationship between activity in dACC, error awareness, and RT. Consistent with this suggestion, we observed faster RT for aware errors than for either unaware errors or correct "Go" responses. dACC activity, however, was not correlated with RT for either aware or unaware errors. As RT is an indirect measure of response conflict, and the EAT is not designed to discriminate between high conflict and low conflict errors, it is possible that this task is not sensitive to the relationship between dACC and conflict (cf. Hughes and Yeung, 2011). We believe, however, that evaluation of a response and awareness of its appropriateness most likely occurs after the response has been executed. In this way, error RT may affect awareness but would not correlate directly with activity in regions believed to be associated with error processing (insula, IPL, dACC).

In the current study, we reported a correlation between activity in the SMA and aware error RT such that higher levels of activity in the SMA were associated with slower aware-error RT. SMC (comprising the SMA and the pre-SMA) is reliably implicated in successful response inhibition (Aron et al., 2007; Simmonds et al., 2008; Sharp et al., 2010). As such, elevated activity in this region associated with aware errors may reflect an insufficient or belated inhibitory response. Garavan and colleagues (2002) reported that clusters of BOLD activity in the SMC were implicated in both successful response inhibition and in commission errors in a Go/No-go task. By combining the fMRI analysis with EEG, the authors demonstrated that commission errors were related to delayed, rather than absent or insufficient, inhibitionrelated activity. In the context of the EAT, as participants become aware of the No-go signal and the drive to inhibit increases, RT will slow but not enough to facilitate inhibition if the prepotent "Go" response was initiated prematurely. This slowing of errorRT is not necessarily a conscious adjustment that depends on awareness of an imminent error, but it seems probable that awareness of the No-go signal is more likely to produce an attempt to inhibit the prepotent response and, should this be unsuccessful, awareness of the error. It has been suggested that the SMC and the dACC serve complimentary roles in performance monitoring: signaling that we are at imminent risk of committing an error or that an error has occurred, respectively (Garavan et al., 2003). It is possible that measures of response conflict indexed by the SMC contribute to the AAC signal by providing an indication of the likelihood of an error and, in this way, dACC activity would covary with response conflict but not correlate directly with errorRT. Alternatively, activity in the SMA and dACC may be driven by activity in another source.

Consistent with previous research using the EAT (Hester et al., 2005, 2009a), regions in the bilateral IPL discriminated aware from unaware errors. Along with the ACC and the insula, the IPL forms part of the frontoparietal control system described by Vincent and colleagues (2008). Seeley and colleagues (2007) argued that within this network the AACC and the insula are associated with the salience of an event and regions in the parietal cortex, including the bilateral IPL, act on events identified as salient. It has been suggested the role of the IPL in cognitive control is to maintain sustained attention on task goals (SinghCurry and Husain, 2009), task parameters (Dosenbach et al., 2006), and response contingencies (Bunge et al., 2003). It is likely that an error constitutes a salient event, and subsequent application of cognitive control may cause participants to re-orient their attention to the task at hand (Coull et al., 1996; SinghCurry and Husain, 2009). In the current study, activity in the IPL was correlated with the post-error adjustment in RT following an aware error such that elevated levels of BOLD activity predicted faster responses on the post-error trial. In the context of the EAT, a salient error may cause an increase in attention to the task parameters or stimulus-response contingencies, thus affecting the speed with which the error awareness response is made on the subsequent trial. 
It must be noted that, although activity in the IPL has previously been associated with error awareness when assessed by the EAT, this activity did not discriminate aware from unaware errors in the anti-saccade task (AST: Klein et al., 2007). It is possible that Klein and colleagues did not observe sensitivity to errors in the IPL due to insufficient statistical power. Alternatively, the EAT may induce a task specific effect due to the response parameters of the Go/No-go task. The EAT requires participants to acknowledge an error with the "error awareness" response rather than the prepotent "Go" response; it is possible that activity in the IPL, pre-SMA, and SMA reflects the intention, preparation, and initiation of the alternative response (Fried et al., 1991; Desmurget and Sirigu, 2009; Desmurget et al., 2009). We believe this to be unlikely, as it would require the preparation of the post-error response to commence during the execution of the erroneous response. Moreover, a previously published study using the EAT (Hester et al., 2005) included a condition that assessed the effect of the additional "awareness" response on BOLD activity in the absence of an error. Only one region in the left middle temporal gyrus showed significant activation for both these odd-ball trials and aware errors, suggesting that the response demands imposed by the task do not account for additional activity observed in the IPL and SMC for aware errors. Unfortunately, this condition was not included in the subsequent studies using the EAT (Hester et al., 2009a, 2012) so we were not able to verify this suggestion with more powerful analysis. In the future, the contribution of the error awareness response to the dACC activity in this paradigm could be assessed by temporally dissociating the awareness response from the commission error itself.

The EAT is not well suited to analyzing post-error behaviors; as discussed above, post-error RT for aware errors is confounded by the requirements of the error awareness response. In error awareness studies using the AST, PES is typically only observed following aware errors (Nieuwenhuis et al., 2001; Wessel et al., 2011) and has been correlated with error-related BOLD activity in the pre-SMA (Klein et al., 2007). Nonetheless, the observation of a small but statistically significant degree of slowing following errors of which the participant was unaware is reliably reported in studies using the EAT and is consistent with reports from two recent studies in which errors were induced by presenting some stimuli below the threshold required for conscious awareness (Pavone et al., 2009; Cohen et al., 2009). Both studies reported PES following aware and unaware errors, but the degree of slowing was greater following errors of which the participants were aware. In the current study, PES following unaware errors was not correlated with the error-related BOLD activity in any of the functionally defined ROI. Using EEG and Granger causality analysis, Cohen and colleagues (2009) revealed increased top-down control over task-relevant sensory regions measured as synchrony between the medial frontal cortex (MFC) and occipital cortex (OCC) following commission errors. The strength of the synchrony between MFC and task-relevant regions was observed to increase following both aware and unaware errors, but was significantly stronger following aware errors. The authors argued that increased synchrony may be the means by which MFC enacts cognitive control by enhancing processing in task-relevant regions, and that this process does not depend on conscious awareness. The BOLD response measured by fMRI does not represent or correlate with all patterns of neural activity, so it may be that increased synchrony leading to PES following unaware errors is not detected in the univariate BOLD response. It is interesting to note that Cohen and colleagues reported no correlation between the degree of PES and the strength of MFC-OCC synchrony; it is possible that increased synchrony is sufficient to induce the less pronounced PES observed following unaware errors but that awareness of the error can induce deliberate remedial strategies over and above these mechanisms, which modulate the nature or degree of post-error behaviors. This suggestion is consistent with the results of Klein and colleagues (2007) in which PES was correlated with error-related activity in the pre-SMA, but only for those errors of which the participant was aware.

It is generally accepted that error awareness is adaptive. Klein and colleagues (2007) reported that the error rate following an aware error was significantly lower than that following an unaware error, suggesting that error awareness improves subsequent performance. In the context of a Go/No-go task, Hester and colleagues (2009b) demonstrated that error-related activity in the dACC, insula, and IPL predicted successful inhibition on the following No-go trial, occurring up to $20 \mathrm{~s}$ later. Although Hester and colleagues (2009b) did not explicitly assess awareness, when considered with the results of the current study, it suggests that error awareness and subsequent inhibition success are subserved by common neural mechanisms. It is surprising, therefore, that we observed no correlation between a participant's level of error awareness and their overall inhibition performance. The absence of a relationship between error awareness and inhibition performance in the EAT has previously been reported by O'Connell and colleagues (2009). It is possible that the application of two inhibition contingencies in the EAT disrupts the relationship between error awareness and subsequent performance, reflecting the role of dACC activity as a reinforcement learning signal (Holroyd and Coles, 2002; Holroyd and Yeung, 2011). This suggestion highlights the need for future studies to assess whether error-related neural activity produces a general, transient increase in the conservatism of responding and a subsequent increase in accuracy, or a context specific, learned adaptation. It would be particularly informative to explore the role of error awareness in these dynamics.

Although models of performance monitoring have consistently implicated the dACC in error processing, the relationship between activity in this region and error awareness has not been reported in previously published fMRI investigations (Hester et al., 2005, 2009a; Klein et al., 2007) and not reliably observed in previously published studies using ERP (Wessel et al., 2011). The increased statistical power afforded by the composite sample used in the current study has shown that error-related BOLD activity in the dACC discriminated aware from unaware errors, consistent with recent reports of error awareness sensitivity in the ERN (Steinhauser and Yeung, 2010; Wessel et al., 2011). Although the results of the present study are consistent with the longstanding belief of a functional relationship between the ERN and BOLD activity in the dACC, Agam and colleagues (2011) have recently suggested that the ERN 
is generated in the PCC. We did not observe error-related BOLD activity in the PCC, so are unable to comment on Agam and colleagues' suggestion that the ERN/PCC response is an errorspecific signal, which is then communicated to the dACC to facilitate behavioral adjustments. The BOLD response is not a direct measure of underlying neural activity and it is possible that the ERN is the result of synchronization of neural populations or the disinhibition of cortical neurons (Agam et al., 2011), phenomena that may not produce significant modulation of the hemodynamic response. It will be necessary to apply multimodal neuroimaging techniques to various error awareness paradigms in order to characterize the role of task and performance factors in the hemodynamic and electrophysiological markers of performance monitoring. Of particular interest will be exploring the influence of the ways in which error awareness is assessed. Those experiments that present some stimuli outside of conscious awareness provide a means of assessing the neural correlates of error awareness without the need of an additional error awareness response that might be vulnerable to failures of memory, task switch costs, or response bias (Woodman, 2010). It remains to be determined whether those errors that occur due to insufficient stimulus representation are as motivationally significant as those that occur due to cognitive failures and whether this mediates error-related neural activity.

\section{REFERENCES}

Agam, Y., Hamalainen, M. S., Lee, A. K., Dyckman, K. A., Friedman, J. S., Isom, M., Makris, N., and Manoach, D. S. (2011). Multimodal neuroimaging dissociates hemodynamic and electrophysiological correlates of error processing. Proc. Natl. Acad. Sci. U.S.A. 108, 17556-17561.

Aron, A. R., Behrens, T. E., Smith, S., Frank, M. J., and Poldrack, R. A. (2007). Triangulating a cognitive control network using diffusionweighted magnetic resonance imaging (MRI) and functional MRI. J. Neurosci. 27, 3743-3752.

Botvinick, M. M., Braver, T. S., Barch, D. M., Carter, C. S., and Cohen, J. D. (2001). Conflict monitoring and cognitive control. Psychol. Rev. 108, 624-652.

Botvinick, M. M., Niv, Y., and Barto, A. C. (2009). Hierarchically organized behaviour and its neural foundations: a reinforcement learning perspective. Cognition 113, 262-280.

Brazil, I. A., de Bruijn, E. R. A., Bulten, B. H., von Borries, A. K. L., van Lankveld, J. J. D. M., Buitelaar, J. K., and Verkes, R. J. (2009). Early and late components of error monitoring in violent offenders with psychopathy. Biol. Psychiatry 65, 137-143.

Brown, J. W., and Braver, T. S. (2005). Learned predictions of error likelihood in the anterior cingulate cortex. Science 307, 1118-1121.

Bunge, S. A., Kahn, I., Wallis, J. D., Miller, E. K., and Wagner, A. D. (2003). Neural circuits subserving the maintenance and retrieval of abstract rules. J. Neurophysiol. 90, 3419-3428.

Burgio-Murphy, A., Klorman, R., Shaywitz, S. E., Fletcher, J. M., Marchione, K. E., Holahan, J., Stuebing, K. K., Thatcher, J. E., and Shaywitz, B. A. (2007). Errorrelated event-related potentials in children with attention-deficit hyperactivity disorder, oppositional defiant disorder, reading disorder, and math disorder. Biol. Psychol. 75, 75-86.

Cohen, M. X., van Gaal, S., Ridderinkhof, K. R., and Lamme, V. A. F. (2009). Unconscious errors enhance prefrontal-occipital oscillatory synchrony. Front. Hum. Neurosci. 3:54. doi: 10.3389/neuro. 09.054.2009

Coles, M. G. H., Scheffers, M. K., and Holroyd, C. B. (2001). Why is there an ERN/Ne on correct trials? Response representations, stimulusrelated components, and the theory of error-processing. Biol. Psychol. 56, 173-189.

Coull, J. T., Frith, C. D., Frackowiak, R. S. J., and Grasby, P. M. (1996). A fronto-parietal network for rapid visual information processing: a PET study of sustained

The current study has demonstrated a network of regions associated with the motoric, cognitive, and motivational components of performance monitoring that discriminated aware from unaware errors. Analyzing the performance of 56 participants revealed error awareness sensitivity in the dACC, but the EAT does not allow us to determine which performance or contextual factors influenced the response of this region and the emergence of error awareness. Our findings are consistent with the suggestion that dACC activity is indirectly related to error awareness and may feed forward into regions directly responsible for consciousness (Wessel et al., 2011) and remedial post-error behavioral adjustments. Error-related activity in the dACC is probably best understood as part of a network in which the constituent regions are differentially sensitive to the demands of the task and the performance context, and that error awareness and error-related behaviors rely on the pattern of activity in this network.

\section{ACKNOWLEDGMENTS}

The authors would like to thank Drs. Mark Bellgrove, Hugh Garavan, Kevin Murphy, and John Foxe for their assistance with the data collection for this study and Mr. Mykalos Byrne for assistance with figures. This work was supported by grants from the Australian Research Council (DP1092852) and National Health and Medical Research Council (1008044) to Robert Hester.

attention. Neuropsychologia 34, 1085-1095.

Cox, R. W. (1996). AFNI: software for analysis and visualization of functional magnetic resonance neuroimages. Comput. Biomed. Res. 29, $162-173$.

Craig, A. D. (2002). How do you feel? Interoception: the sense of the physiological condition of the body. Nat. Rev. Neurosci. 3, 655-666.

Craig, A. D. (2009). How do you feel-now? The anterior insula and human awareness. Nat. Rev. Neurosci. 10, 59-70.

Critchley, H. D. (2005). Neural mechanisms of autonomic, affective, and cognitive integration. J. Comp. Neurol. 493, 154-166.

Critchley, H. D., Mathias, C. J., and Dolan, R. J. (2002). Fear conditioning in humans: the influence of awareness and autonomic arousal on functional neuroanatomy. Neuron 33, 653-663.

Critchley, H. D., Melmed, R. N., Featherstone, E., Mathias, C. J., and Dolan, R. J. (2001). Brain activity during biofeedback relaxation: a functional neuroimaging investigation. Brain 124, 1003-1012.

Critchley, H. D., Tang, J., Glaser, D., Butterworth, B., and Dolan, R. J. (2005). Anterior cingulate activity during error and autonomic response. Neuroimage 27, 885-895.

Critchley, H. D., Wiens, S., Rotshtein, P., Ohman, A., and Dolan, R. J.
(2004). Neural systems supporting interoceptive awareness. Nat. Neurosci. 7, 189-195.

Critchley, H. D., Elliott, R., Mathias, C. J., and Dolan, R. J. (2000) Neural activity relating to generation and representation of galvanic skin conductance responses: a functional magnetic resonance imaging study. J. Neurosci. 20, 3033-3040.

Debener, S., Ullsperger, M., Siegel, M., Fiehler, K., von Cramon, D. Y., and Engel, A. K. (2005). Trial-by-trial coupling of concurrent electroencephalogram and functional magnetic resonance imaging identifies the dynamics of performance monitoring. J. Neurosci. 25, 11730-11737.

Dehaene, S., Posner, M. I., and Tucker, D. M. (1994). Localization of a neural system for error detection and compensation. Psychol. Sci. 5, 303-305.

Dehaene, S., Sergent, C., and Changeux, J. P. (2003). A neuronal network model linking subjective reports and objective physiological data during conscious perception. Proc. Natl. Acad. Sci. U.S.A. 100, 8520-8525.

Desmurget, M., Reilly, K. T., Richard, N., Szathmari, A., Mottolese, C., and Sirigu, A. (2009). Movement intention after parietal cortex stimulation in humans. Science 324, 811-813.

Desmurget, M., and Sirigu, A. (2009). A parietal-premotor network for 
movement intention and motor awareness. Trends Cogn. Sci. 13, 411-419.

Dosenbach, N. U. F., Visscher, K. M., Palmer, E. D., Miezin, F. M., Wenger, K. K., Kang, H. C., Burgund, E. D., Grimes, A. L., Schlagger, B. L., and Petersen, S. E. (2006). A core system for the implementation of task sets. Neuron 50, 799-812.

Endrass, T., Reuter, B., and Kathmann, N. (2005). Effects of error awareness on error related brain potentials in an antisaccade task. J. Psychophysiol. 19, 114.

Endrass, T., Reuter, B., and Kathmann, N. (2007). ERP correlates of conscious error recognition: aware and unaware errors in an antisaccade task. Eur. J. Neurosci. 26, 1714-1720.

Falkenstein, M., Hohnsbein, J., Hoormann, J., and Blanke, L. (1991). Effects of crossmodal divided attention on late ERP components. II. Error processing in choice reaction tasks. Electroencephalogr. Clin. Neurophysiol. 78, 447-455.

Franken, I. H. A., Rassin, E., and Muris, P. (2007). The assessment of anhedonia in clinical and non-clinical populations: further validation of the Snaith-Hamilton Pleasure Scale (SHAPS). J. Affect. Disord. 99, 83-89.

Fried, I., Katz, A., Mccarthy, G., Sass, K. J., Williamson, P., Spencer, S. S., and Spencer, D. D. (1991). Functional organization of human supplementary motor cortex studied by electrical-stimulation. J. Neurosci. 11, 3656-3666.

Garavan, H., Ross, T. J., Kaufman, J., and Stein, E. A. (2003). A midline dissociation between error-processing and responseconflict monitoring. Neuroimage 20, 1132-1139.

Garavan, H., Ross, T. J., Murphy, K., Roche, R. A. P., and Stein, E. A. (2002). Dissociable executive functions in the dynamic control of behavior: Inhibition, error detection, and correction. Neuroimage 17, 1820-1829.

Halgren, E., Boujon, C., Clarke, J., and Wang, C. (2002). Rapid distributed fronto-parieto-occipital processing stages during working memory in humans. Cereb. Cortex 12, 710-728.

Hester, R., Foxe, J. J., Molholm, S., Shpaner, M., and Garavan, H. (2005). Neural mechanisms involved in error processing: a comparison of errors made with and without awareness. Neuroimage 27, 602-608.

Hester, R., Madeley, J., Murphy, K., and Mattingley, J. B. (2009a). Learning from errors: error-related neural activity predicts improvements in future inhibitory control performance. J. Neurosci. 29, 7158-7165.

Hester, R., Nestor, L., and Garavan, H. (2009b). Impaired error awareness and anterior cingulate cortex hypoactivity in chronic cannabis users. Neuropsychopharmacology 34, 2450-2458.

Hester, R., Nandam, S., O’Connell, R., Wagner, J., Strudwick, M., Nathan, P., Mattingley, J., and Bellgrove, M. (2012). Neurochemical enhancement of conscious error awareness. J. Neurosci. 32, 2619-2627.

Hester, R., Simoes-Franklin, C., and Garavan, H. (2007). Post-error behavior in active cocaine users: poor awareness of errors in the presence of intact performance adjustments. Neuropsychopharmacology 32, 1974-1984.

Holroyd, C. B., and Coles, M. G. H. (2002). The neural basis. of human error processing: reinforcement learning, dopamine, and the errorrelated negativity. Psychol. Rev. 109, 679-709.

Holroyd, C. B., Nieuwenhuis, S., Yeung, N., Nystrom, L., Mars, R. B., Coles, M. G. H., and Cohen, J. D. (2004) Dorsal anterior cingulate cortex shows fMRI response to internal and external error signals. Nat. Neurosci. 7, 497-498.

Holroyd, C. B., and Yeung, N. (2011). "An integrative theory of anterior cingulate cortex function: Option selection in hierarchical reinforcement learning," in The Neural Basis of Motivational and Cognitive Control, eds R. B. Mars, J. Sallet, M. F. S. Rushworth, and N. Yeung (Cambridge, MA: MIT Press), 333-349.

Hughes, G., and Yeung, N. (2011). Dissociable correlates of response conflict and error awareness in error-related brain activity. Neuropsychologia 49, 405-415.

Keppel, G. (1991). Design and Analysis: A Researcher's Handbook. New Jersey, Englewood Cliffs NJ: Prentice Hall.

Kerns, J. G., Cohen, J. D., MacDonald, A. W., Cho, R. Y., Stenger, V. A., and Carter, C. S. (2004). Anterior Cingulate conflict monitoring and adjustments in control. Science 303, 1023-1026.

Klein, T. A., Endrass, T., Kathmann, N., Neumann, J., von Cramon, D. Y., and Ullsperger, M. (2007). Neural correlates of error awareness. Neuroimage 34, 1774-1781.

Maier, M., Steinhauser, M., and Hubner, R. (2008). Is the errorrelated negativity amplitude related to error detectability? Evidence from effects of different error types. J. Cogn. Neurosci. 20, 2263-2273.

Mathalon, D. H., Bennett, A., Askari, N., Gray, E. M., Rosenbloom, M. J., and Ford, J. M. (2003). Responsemonitoring dysfunction in aging and Alzheimer's disease: an eventrelated potential study. Neurobiol. Aging 24, 675-685.

Mayr, U. (2004). Conflict, consciousness, and control. Trends Cogn. Sci. 8, 145-148.

Morris, S. E., Yee, C. M., and Nuechterlein, K. H. (2006). Electrophysiological analysis of error monitoring in schizophrenia. J. Abnorm. Psychol. 115, 239-250.

Murphy, K., and Garavan, H. (2005) Deriving the optimal number of events for an event-related fMR study based on the spatial extent of activation. Neuroimage 27, 771-777.

Nieuwenhuis, S., Ridderinkhof, K. R., Blow, J., Band, G. P. H., and Kok, A. (2001). Error-related brain potentials are differentially related to awareness of response errors: evidence from an antisaccade task. Psychophysiology 38, 752-760.

O'Connell, R. G., Bellgrove, M. A. Dockree, P. M., Lau, A., Hester, R., Garavan, H., Fitzgerald, M., Foxe, J. J., and Robertson, I. H. (2009). The neural correlates of deficient error awareness in attention-deficit hyperactivity disorder (ADHD). Neuropsychologia 47, 1149-1159.

O'Connell, R. G., Dockree, P. M. Bellgrove, M. A., Kelly, S. P., Hester, R., Garavan, H., Robertson, I. H., and Foxe, J. J. (2007). The role of cingulate cortex in the detection of errors with and without awareness: a high-density electrical mapping study. Eur. J. Neurosci. 25, 2571-2579.

Overbeek, T. J. M., Nieuwenhuis, S., and Ridderinkhof, K. R. (2005). Dissociable components of error processing - On the functional significance of the Pe Vis-a-vis the ERN/Ne. J. Psychophysiol. 19, 319-329.

Pavone, E. F., Marzi, C. A., and Girelli, M. (2009). Does subliminal visual perception have an errormonitoring system? Eur. J. Neurosci. 30, 1424-1431.

Rabbitt, P. M. A. (1966). Errors and error correction in choice-response tasks. J. Exp. Psychol. 71, 264-272.

Ridderinkhof, K. R., Ramautar, J. R., and Wijnen, J. G. (2009). To P(E) or not to $\mathrm{P}(\mathrm{E})$ : a $\mathrm{P}$-like ERP component reflecting the processing of response errors. Psychophysiology 46, 531-538.

Ridderinkhof, K. R., Ullsperger, M., Crone, E. A., and Nieuwenhuiss, S. (2004). The role of the medial frontal cortex in cognitive control. Science 306, 443-447.

Scheffers, M. K., and Coles, M. G. H. (2000). Performance monitoring in a confusing world: errorrelated brain activity, judgments of response accuracy, and types of errors. J. Exp. Psychol. Hum. Percept. Perform. 26, 141-151.

Seeley, W. W., Menon, V., Schatzberg, A. F., Keller, J., Glover, G. H., Kenna, H., Reiss, A. L., and Greicius, M. D. (2007). Dissociable intrinsic connectivity networks for salience processing and executive control. J. Neurosci. 27, 2349-2356.

Shalgi, S., O'Connell, R. G., Deouell, L. Y., and Robertson, I. H. (2007) Absent minded but accurate: delaying responses increases accuracy but decreases error awareness. Exp. Brain Res. 182, 119-124.

Sharp, D. J., Bonnelle, V., De Boissezon, X., Beckmann, C. F., James, S. G., Patel, M. C., and Mehta, M. A. (2010). Distinct frontal systems for response inhibition, attentional capture, and error processing. Proc. Natl. Acad. Sci. U.S.A. 107, 6106-6111.

Simmonds, D. J., Pekar, J. J., and Mostofsky, S. H. (2008). Metaanalysis of Go/No-go tasks demonstrating that fMRI activation associated with response inhibition is task-dependent Neuropsychologia 46, 224-232.

Singh-Curry, V., and Husain, M. (2009). The functional role of the inferior parietal lobe in the dorsal and ventral stream dichotomy. Neuropsychologia 47, 1434-1448.

Soltani, M., and Knight, R. T. (2000). Neural origins of the P300. Crit. Rev. Neurobiol. 14, 199-224.

Steinhauser, M., and Yeung, N. (2010). Decision processes in human performance monitoring. J. Neurosci. 30, 15643-15653

Ullsperger, M., and von Cramon, D. Y. (2001). Subprocesses of performance monitoring: a dissociation of error processing and response competition revealed by event-related fMRI and ERPs. Neuroimage 14, 1387-1401.

Ullsperger, M., Harsay, H. A., Wessel, J. R., and Ridderinkhof, K. R. (2010). Conscious perception of errors and its relation to the anterior insula. Brain Struct. Funct. 214, 629-643.

Vincent, J. L., Kahn, I., Snyder, A. Z., Raichle, M. E., and Buckner, 
R. L. (2008). Evidence for a frontoparietal control system revealed by intrinsic functional connectivity. J. Neurophysiol. 100, 3328-3342.

Wang, C. M., Ulbert, I., Schomer, D. L., Marinkovic, K., and Halgren, E. (2005). Responses of human anterior cingulate cortex microdomains to error detection, conflict monitoring, stimulus-response mapping, familiarity, and orienting. J. Neurosci. 25, 604-613.

Wessel, J. R., Danielmeier, C., and Ullsperger, M. (2011). Error awareness revisited: accumulation of multimodal evidence from central and autonomic nervous systems. J. Cogn. Neurosci. 23, 3021-3036.

Woodman, G. F. (2010). Masked targets trigger event-related potentials index shifts of attention but not error detection. Psychophysiology 47, 410-414.

Yeung, N., Botvinick, M. M., and Cohen, J. D. (2004). The neural basis of error detection: conflict monitoring and the error-related negativity. Psychol. Rev. 111, 931-959.

Conflict of Interest Statement: The authors declare that the research was conducted in the absence of any commercial or financial relationships that could be construed as a potentia conflict of interest.

Received: 31 January 2012; accepted: 31 May 2012; published online: 19 June 2012.
Citation: Orr $C$ and Hester $R$ (2012) Error-related anterior cingulate cortex activity and the prediction of conscious error awareness. Front. Hum. Neurosci. 6:177. doi: 10.3389/fnhum.2012.00177 Copyright (c) 2012 Orr and Hester. This is an open-access article distributed under the terms of the Creative Commons Attribution Non Commercial License, which permits non-commercial use, distribution, and reproduction in other forums, provided the original authors and source are credited. 


\section{APPENDIX}

In order to generate the constituent maps for error-related activity associated with aware and unaware errors, group activation maps were determined with one-sample $t$-tests against the null hypothesis of zero event-related activation changes (i.e., no change relative to baseline). Significant voxels within group maps passed a voxelwise statistical threshold $(p \leq 0.0001)$ and were required to be part of a larger $84 \mu \mathrm{l}$ cluster of contiguous significant voxels.
The event-related functional analysis of aware errors identified nine clusters that showed significantly greater activity associated with aware errors than with correct go-trial responses (Table A1).

The event-related functional analysis of unaware errors identified eight clusters that showed significantly greater activity associated with unaware errors than with correct go-trial responses (Table A2).

Table A1 | Regions that showed significantly greater BOLD signal change for aware errors than for correct go-trial responses.

\begin{tabular}{|c|c|c|c|c|c|c|}
\hline Structure & Volume $\mu \mathrm{I}$ & Brodmann area & HS & \multicolumn{3}{|c|}{ Center of mass (MNI Coordinates) } \\
\hline Inferior parietal & 7776 & 40 & L & -48 & -40 & 46 \\
\hline Inferior parietal & 6529 & 40 & $\mathrm{R}$ & 47 & -49 & 40 \\
\hline $\mathrm{dACC}$ & 4878 & 32 & $\mathrm{~L}$ & 0 & 9 & 43 \\
\hline Insula & 3020 & 13 & $\mathrm{R}$ & 44 & 13 & 4 \\
\hline Extrastriate cortex & 118 & 7 & $L$ & -9 & -77 & 31 \\
\hline Precentral gyrus/SMA & 116 & $4 / 6$ & $L$ & -28 & -27 & 70 \\
\hline Middle frontal gyrus & 84 & 9 & $\mathrm{R}$ & 39 & 12 & 46 \\
\hline
\end{tabular}

Positive values for $x, y$, and $z$ coordinates denote, respectively, locations that are right, posterior, and superior relative to the anterior commissure. Significance test results indicate areas of increased activation associated with aware relative to unaware errors assessed using t-test.

Table A2 | Regions that showed significantly greater BOLD signal change for unaware errors than for correct go-trial responses.

\begin{tabular}{|c|c|c|c|c|c|c|}
\hline Structure & Volume $\mu \mathrm{l}$ & Brodmann area & HS & \multicolumn{3}{|c|}{ Center of mass (MNI Coordinates) } \\
\hline Middle occipital gyrus & 217 & 19 & $\mathrm{~L}$ & -38 & -78 & -2 \\
\hline Lingual gyrus & 164 & 18 & $\mathrm{R}$ & 17 & -81 & -14 \\
\hline Middle occipital gyrus & 127 & 19 & $\mathrm{R}$ & 34 & -76 & -15 \\
\hline Superior temporal gyrus & 96 & 41 & $\mathrm{R}$ & 52 & -24 & 7 \\
\hline Caudate & 88 & & $\mathrm{R}$ & 13 & 22 & 7 \\
\hline
\end{tabular}

Positive values for $x, y$, and $z$ coordinates denote, respectively, locations that are right, posterior, and superior relative to the anterior commissure. Significance test results indicate areas of increased activation associated with aware relative to unaware errors assessed using t-test. 